Скопје, Македонија

\title{
INEQUALITIES FOR THE DUAL RELATIVE OPERATOR ENTROPY
}

\author{
SILVESTRU SEVER DRAGOMIR
}

\begin{abstract}
In this paper, we introduce the concept of dual relative entropy defined by

$$
D(A \mid B):=A^{1 / 2}\left(A^{-1 / 2} B A^{-1 / 2} \ln \left(A^{-1 / 2} B A^{-1 / 2}\right)\right) A^{1 / 2}
$$

for positive invertible operators $A$ and $B$ and establish various upper and lower bounds for the error operator in approximating the $D(A \mid B)$ by

$$
\frac{m \ln m}{M-m}(M A-B)+\frac{M \ln M}{M-m}(B-m A)
$$

under the natural assumption $m A \leq B \leq M A$ for some $m, M$ with $0<m<M$. Applications for the operator entropy are also given. Some trace inequalities are derived as well.
\end{abstract}

Kamei and Fujii [8], [9] defined the relative operator entropy $S(A \mid B)$, for positive invertible operators $A$ and $B$, by

$$
S(A \mid B):=A^{\frac{1}{2}}\left(\ln A^{-\frac{1}{2}} B A^{-\frac{1}{2}}\right) A^{\frac{1}{2}},
$$

which is a relative version of the operator entropy considered by NakamuraUmegaki [16].

In general, we can define for positive operators $A, B$

$$
S(A \mid B):=s-\lim _{\varepsilon \rightarrow 0+} S(A+\varepsilon I \mid B)
$$

if it exists, here $I$ is the identity operator.

For the entropy function $\eta(t)=-t \ln t$, the operator entropy has the following expression:

$$
\eta(A)=-A \ln A=S(A \mid I) \geq 0
$$

for positive contraction $A$. This shows that the relative operator entropy (1) is a relative version of the operator entropy.

2010 Mathematics Subject Classification. Primary: 47A63 Secondary: 47A30.

Key words and phrases. Young's inequality, Arithmetic mean-Geometric mean inequality, Relative Operator entropy, Operator entropy. 
Following [10, p. 149-p. 155], we recall some important properties of relative operator entropy for $A$ and $B$ positive invertible operators:

(i) We have the equalities

$$
S(A \mid B)=-A^{1 / 2}\left(\ln A^{1 / 2} B^{-1} A^{1 / 2}\right) A^{1 / 2}=B^{1 / 2} \eta\left(B^{-1 / 2} A B^{-1 / 2}\right) B^{1 / 2}
$$

(ii) We have the inequalities

$$
S(A \mid B) \leq A(\ln \|B\|-\ln A) \text { and } S(A \mid B) \leq B-A ;
$$

(iii) For any $C, D$ positive invertible operators we have that

$$
S(A+B \mid C+D) \geq S(A \mid C)+S(B \mid D) ;
$$

(iv) If $B \leq C$ then

$$
S(A \mid B) \leq S(A \mid C)
$$

(v) If $B_{n} \downarrow B$ then

$$
S\left(A \mid B_{n}\right) \downarrow S(A \mid B) ;
$$

(vi) For $\alpha>0$ we have

$$
S(\alpha A \mid \alpha B)=\alpha S(A \mid B) ;
$$

(vii) For every operator $T$ we have

$$
T^{*} S(A \mid B) T \leq S\left(T^{*} A T \mid T^{*} B T\right) .
$$

The relative operator entropy is jointly concave, namely, for any positive invertible operators $A, B, C, D$ we have

$$
S(t A+(1-t) B \mid t C+(1-t) D) \geq t S(A \mid C)+(1-t) S(B \mid D)
$$

for any $t \in[0,1]$.

For other results on the relative operator entropy see [6], [12], [13], [15] and [17].

In the recent paper [5] we have obtained amongst other the following result in approximating the relative operator entropy $S(A \mid B)$ by some simpler quantity:

Theorem 1. Let $A, B$ be two positive invertible operators such that the condition

$$
m A \leq B \leq M A,
$$

for some $m, M$ with $0<m<M$, is valid, then we have

$$
\begin{aligned}
& \frac{1}{2 M^{2}}(B-m A) A^{-1}(M A-B) \\
& \leq S(A \mid B)-\frac{\ln m}{M-m}(M A-B)-\frac{\ln M}{M-m}(B-m A) \\
& \leq \frac{1}{2 m^{2}}(B-m A) A^{-1}(M A-B) .
\end{aligned}
$$


In particular, we have the following result for the operator entropy:

Corollary 1. Assume that $p I \leq C \leq P I$ for some constants $p, P$ with $0<p<P$. Then we have for operator entropy $\eta(C)=-C \ln C$ that

$$
\begin{aligned}
& \frac{p}{2 P}(I P-C) C^{-1}(C-I p) \\
& \leq \eta(C)+\frac{P \ln P}{P-p}(C-p I)+\frac{p \ln p}{P-p}(P I-C) \\
& \leq \frac{P}{2 p}(I P-C) C^{-1}(C-I p) .
\end{aligned}
$$

Taking into account the above, we can introduce the concept of dual relative entropy defined by

$$
D(A \mid B):=A^{1 / 2}\left(A^{-1 / 2} B A^{-1 / 2} \ln \left(A^{-1 / 2} B A^{-1 / 2}\right)\right) A^{1 / 2}
$$

for positive invertible operators $A$ and $B$.

Observe that, if we replace in (2) $B$ with $A$, then we get

$$
\begin{aligned}
S(B \mid A) & =A^{1 / 2} \eta\left(A^{-1 / 2} B A^{-1 / 2}\right) A^{1 / 2} \\
& =A^{1 / 2}\left(-A^{-1 / 2} B A^{-1 / 2} \ln \left(A^{-1 / 2} B A^{-1 / 2}\right)\right) A^{1 / 2},
\end{aligned}
$$

therefore we have

$$
A^{1 / 2}\left(A^{-1 / 2} B A^{-1 / 2} \ln \left(A^{-1 / 2} B A^{-1 / 2}\right)\right) A^{1 / 2}=-S(B \mid A)
$$

for positive invertible operators $A$ and $B$, which shows that the dual relative entropy has the following representation in terms of the relative entropy:

$$
D(A \mid B)=-S(B \mid A)
$$

for positive invertible operators $A$ and $B$. It is also well know that, in general $S(A \mid B)$ is not equal to $S(B \mid A)$.

Motivated by the above results, we establish in this paper some error bounds in approximation of the dual relative entropy $D(A \mid B)$ with the simpler quantity

$$
\frac{m \ln m}{M-m}(M A-B)+\frac{M \ln M}{M-m}(B-m A)
$$

under the natural assumptions (3) for the operators $A$ and $B$, namely $m A \leq$ $B \leq M A$, for some $m, M$ with $0<m<M$. For this purpose, we use some scalar inequalities for convex functions from [2], [3] and [4]. Applications for the operator entropy $\eta(C)=-C \ln C=S(C \mid I)$ under the natural assumption $p I \leq C \leq P I$ for some constants $p, P$ with $0<p<P$, are also provided. 


\section{Absolute Value Upper and Lower Bounds}

With the assumption that the operators $A$ and $B$ satisfy the condition $m A \leq B \leq M A$, for some $m, M$ with $0<m<M$, define the error operator

$$
E_{m, M}(A, B):=\frac{m \ln m}{M-m}(M A-B)+\frac{M \ln M}{M-m}(B-m A)-D(A \mid B),
$$

which represent the error in approximating the dual relative operator entropy by the operator from (7)

The next result provided some upper and lower bounds for the error operator $E_{m, M}(A, B)$.

Theorem 2. Let $A, B$ be two positive invertible operators such that the condition (3) is valid, then we have

$$
\begin{aligned}
& 2\left(\frac{1}{2} A-\frac{1}{M-m} A^{1 / 2}\left|A^{-1 / 2}\left(B-\frac{m+M}{2} A\right) A^{-1 / 2}\right| A^{1 / 2}\right) K(m, M) \\
& \leq E_{m, M}(A, B) \\
& \leq 2\left(\frac{1}{2} A+\frac{1}{M-m} A^{1 / 2}\left|A^{-1 / 2}\left(B-\frac{m+M}{2} A\right) A^{-1 / 2}\right| A^{1 / 2}\right) K(m, M),
\end{aligned}
$$

where

$$
\begin{aligned}
K(m, M) & :=\left[\frac{m \ln m+M \ln M}{2}-\left(\frac{m+M}{2}\right) \ln \left(\frac{m+M}{2}\right)\right] \\
& =\ln \left(\frac{G\left(m^{m}, M^{M}\right)}{[A(m, M)]^{A(m, M)}}\right)
\end{aligned}
$$

and $G(a, b):=\sqrt{a b}$ is the geometric mean while $A(a, b):=\frac{a+b}{2}$ is the arithmetic mean of positive numbers $a, b$.

Proof. Recall the following result obtained by the author in 2006 [2] that provides a refinement and a reverse for the weighted Jensen's discrete inequality:

$$
\begin{aligned}
& \min _{j \in\{1,2, \ldots, n\}}\left\{p_{j}\right\}\left[\frac{1}{n} \sum_{j=1}^{n} \Phi\left(x_{j}\right)-\Phi\left(\frac{1}{n} \sum_{j=1}^{n} x_{j}\right)\right] \\
& \leq \frac{1}{P_{n}} \sum_{j=1}^{n} p_{j} \Phi\left(x_{j}\right)-\Phi\left(\frac{1}{P_{n}} \sum_{j=1}^{n} p_{j} x_{j}\right) \\
& \leq n \max _{j \in\{1,2, \ldots, n\}}\left\{p_{j}\right\}\left[\frac{1}{n} \sum_{j=1}^{n} \Phi\left(x_{j}\right)-\Phi\left(\frac{1}{n} \sum_{j=1}^{n} x_{j}\right)\right],
\end{aligned}
$$


where $\Phi: C \rightarrow \mathbb{R}$ is a convex function defined on convex subset $C$ of the linear space $X,\left\{x_{j}\right\}_{j \in\{1,2, \ldots, n\}}$ are vectors in $C$ and $\left\{p_{j}\right\}_{j \in\{1,2, \ldots, n\}}$ are nonnegative numbers with $P_{n}=\sum_{j=1}^{n} p_{j}>0$. For $n=2$, we deduce from (10) that

$$
\begin{aligned}
& 2 r\left[\frac{\Phi(x)+\Phi(y)}{2}-\Phi\left(\frac{x+y}{2}\right)\right] \\
& \leq \nu \Phi(x)+(1-\nu) \Phi(y)-\Phi[\nu x+(1-\nu) y] \\
& \leq 2 R\left[\frac{\Phi(x)+\Phi(y)}{2}-\Phi\left(\frac{x+y}{2}\right)\right]
\end{aligned}
$$

for any $x, y \in \mathbb{R}$ and $\nu \in[0,1]$, where $r=\min \{\nu, 1-\nu\}$ and $R=$ $\max \{\nu, 1-\nu\}$.

Now, if we take in (11) the convex function $\Phi(t)=t \ln t, t>0$, then we get

$$
\begin{aligned}
& 2 r\left[\frac{x \ln x+y \ln y}{2}-\left(\frac{x+y}{2}\right) \ln \left(\frac{x+y}{2}\right)\right] \\
& \leq \nu x \ln x+(1-\nu) y \ln y-[\nu x+(1-\nu) y] \ln [\nu x+(1-\nu) y] \\
& \leq 2 R\left[\frac{x \ln x+y \ln y}{2}-\left(\frac{x+y}{2}\right) \ln \left(\frac{x+y}{2}\right)\right]
\end{aligned}
$$

for any $x, y>0$ and $\nu \in[0,1]$.

This is an inequality of interest in itself as well.

Now, if we take in (12) $x=m, y=M$ and $\nu=\frac{M-u}{M-m} \in[0,1]$ with $u \in[m, M]$ then we get

$$
\begin{aligned}
& 2 \min \left\{\frac{M-u}{M-m}, \frac{u-m}{M-m}\right\} \\
& \times\left[\frac{m \ln m+M \ln M}{2}-\left(\frac{m+M}{2}\right) \ln \left(\frac{m+M}{2}\right)\right] \\
& \leq \frac{M-u}{M-m} m \ln m+\frac{u-m}{M-m} M \ln M-u \ln u \\
& \leq 2 \max \left\{\frac{M-u}{M-m}, \frac{u-m}{M-m}\right\} \\
& \times\left[\frac{m \ln m+M \ln M}{2}-\left(\frac{m+M}{2}\right) \ln \left(\frac{m+M}{2}\right)\right] .
\end{aligned}
$$

Since

$$
\min \left\{\frac{M-u}{M-m}, \frac{u-m}{M-m}\right\}=\frac{1}{2}-\left|\frac{u-\frac{m+M}{2}}{M-m}\right|
$$

and

$$
\max \left\{\frac{M-u}{M-m}, \frac{u-m}{M-m}\right\}=\frac{1}{2}+\left|\frac{u-\frac{m+M}{2}}{M-m}\right| \text {, }
$$


then from (13) we have

$$
\begin{aligned}
& 2\left(\frac{1}{2}-\frac{1}{M-m}\left|u-\frac{m+M}{2}\right|\right) K(m, M) \\
& \leq \frac{M-u}{M-m} m \ln m+\frac{u-m}{M-m} M \ln M-u \ln u \\
& \leq 2\left(\frac{1}{2}+\frac{1}{M-m}\left|u-\frac{m+M}{2}\right|\right) K(m, M)
\end{aligned}
$$

for any $u \in[m, M]$.

Using the continuous functional calculus we have from (14) that

$$
\begin{aligned}
& 2\left(\frac{1}{2} I-\frac{1}{M-m}\left|X-\frac{m+M}{2} I\right|\right) K(m, M) \\
& \leq m \ln m \frac{M I-X}{M-m}+M \ln M \frac{X-m I}{M-m}-X \ln X \\
& \leq 2\left(\frac{1}{2} I+\frac{1}{M-m}\left|X-\frac{m+M}{2} I\right|\right) K(m, M)
\end{aligned}
$$

for any selfadjoint operator $X$ with the property that $m I \leq X \leq M I$.

Multiplying both sides of (3) by $A^{-1 / 2}$ we get

$$
m I \leq A^{-1 / 2} B A^{-1 / 2} \leq M I
$$

and by replacing $X$ by $A^{-1 / 2} B A^{-1 / 2}$ in (15) we obtain

$$
\begin{aligned}
& 2\left(\frac{1}{2} I-\frac{1}{M-m}\left|A^{-1 / 2} B A^{-1 / 2}-\frac{m+M}{2} I\right|\right) K(m, M) \\
& \leq m \ln m \frac{M I-A^{-1 / 2} B A^{-1 / 2}}{M-m}+M \ln M \frac{A^{-1 / 2} B A^{-1 / 2}-m I}{M-m} \\
& -A^{-1 / 2} B A^{-1 / 2} \ln \left(A^{-1 / 2} B A^{-1 / 2}\right) \\
& \leq 2\left(\frac{1}{2} I+\frac{1}{M-m}\left|A^{-1 / 2} B A^{-1 / 2}-\frac{m+M}{2} I\right|\right) K(m, M) .
\end{aligned}
$$

Multiplying both sides of (16) by $A^{1 / 2}$ we get the desired result (9).

Remark 1. One can observe that the inequalities (10) are a simple consequence of Theorem 1, p.717 from [14]. Similar scalar inequalities as those in the proof of the theorem were obtained in [1] and [11].

Remark 2. If $A$ and $B$ commute, then

$$
\begin{gathered}
A^{1 / 2}\left|A^{-1 / 2}\left(B-\frac{m+M}{2} A\right) A^{-1 / 2}\right| A^{1 / 2}=\left|B-\frac{m+M}{2} A\right|, \\
S(B \mid A)=B(\ln A-\ln B)
\end{gathered}
$$


INEQUALITIES FOR THE DUAL RELATIVE OPERATOR ENTROPY

and by (9) we have

$$
\begin{aligned}
& (0 \leq) 2\left(\frac{1}{2} A-\frac{1}{M-m}\left|B-\frac{m+M}{2} A\right|\right) K(m, M) \\
& \leq \frac{m \ln m}{M-m}(M A-B)+\frac{M \ln M}{M-m}(B-m A)-B(\ln B-\ln A) \\
& \leq 2\left(\frac{1}{2} A+\frac{1}{M-m}\left|B-\frac{m+M}{2} A\right|\right) K(m, M) .
\end{aligned}
$$

The above result can be applied for the operator entropy

$$
\eta(C)=-C \ln C=S(C \mid I)
$$

as follows:

Corollary 2. Assume that $p I \leq C \leq P I$ for some $p, P$ with $0<p<P$. Then we have that

$$
\begin{aligned}
& (0 \leq) 2\left(\frac{1}{2} I-\frac{1}{P-p}\left|C-\frac{p+P}{2} I\right|\right) K(p, P) \\
& \leq \frac{p \ln p}{P-p}(P I-C)+\frac{P \ln P}{P-p}(C-p I)+\eta(C) \\
& \leq 2\left(\frac{1}{2} I+\frac{1}{P-p}\left|C-\frac{p+P}{2} I\right|\right) K(p, P) .
\end{aligned}
$$

\section{An Upper Bound in Terms of Logarithm}

We have the following inequality of interest for convex functions, see for instance [3]:

Lemma 1. Let $f: I \subset \mathbb{R} \rightarrow \mathbb{R}$ be a convex function on the interval $I$, $a, b \in \stackrel{\circ}{I}$, the interior of $I$ and $\nu \in[0,1]$. Then

$$
\begin{aligned}
0 \leq(1-\nu) f(a)+\nu f(b)-f & (1-\nu) a+\nu b) \\
& \leq \nu(1-\nu)(b-a)\left[f_{-}^{\prime}(b)-f_{+}^{\prime}(a)\right] .
\end{aligned}
$$

In particular, we have

$$
0 \leq \frac{f(a)+f(b)}{2}-f\left(\frac{a+b}{2}\right) \leq \frac{1}{4}(b-a)\left[f_{-}^{\prime}(b)-f_{+}^{\prime}(a)\right] .
$$

The constant $\frac{1}{4}$ is best possible in both inequalities from (20).

We can state the following result: 
Theorem 3. Let $A, B$ be two positive invertible operators such that the condition (3) is valid, then we have

$$
\begin{aligned}
(0 \leq) E_{m, M}(A, B) & \leq \frac{\ln M-\ln m}{M-m}(B-m A) A^{-1}(M A-B) \\
& \leq \frac{1}{4}(M-m)(\ln M-\ln m) A .
\end{aligned}
$$

Proof. If we consider the convex function $f(t)=t \ln t, t>0$, then $f^{\prime}(t)=$ $\ln t+1$ and by (19) we have

$$
\begin{aligned}
0 & \leq(1-\nu) a \ln a+\nu b \ln b-((1-\nu) a+\nu b) \ln ((1-\nu) a+\nu b) \\
& \leq \nu(1-\nu)(b-a)(\ln b-\ln a)
\end{aligned}
$$

for any $a, b>0$ and and $\nu \in[0,1]$.

On applying the inequality $(22)$ on the interval $[m, M]$ and for $\nu=$ $\frac{x-m}{M-m} \in[0,1]$ with $x \in[m, M]$ then we get

$$
\begin{aligned}
0 & \leq m \ln m \frac{M-x}{M-m}+M \ln M \frac{x-m}{M-m}-x \ln x \\
& \leq \frac{(x-m)(M-x)}{M-m}(\ln M-\ln m) \leq \frac{1}{4}(M-m)(\ln M-\ln m) .
\end{aligned}
$$

Using the continuous functional calculus we have from (23) that

$$
\begin{aligned}
0 & \leq m \ln m \frac{M I-X}{M-m}+M \ln M \frac{X-m I}{M-m}-X \ln X \\
& \leq(\ln M-\ln m) \frac{(X-m I)(M-X I)}{M-m} \leq \frac{1}{4}(M-m)(\ln M-\ln m) I
\end{aligned}
$$

for any selfadjoint operator $X$ with the property that $m I \leq X \leq M I$.

By replacing $X$ by $A^{-1 / 2} B A^{-1 / 2}$ in (15) we get

$$
\begin{aligned}
0 & \leq m \ln m \frac{M I-A^{-1 / 2} B A^{-1 / 2}}{M-m}+M \ln M \frac{A^{-1 / 2} B A^{-1 / 2}-m I}{M-m} \\
& -A^{-1 / 2} B A^{-1 / 2} \ln \left(A^{-1 / 2} B A^{-1 / 2}\right) \\
& \leq(\ln M-\ln m) \frac{\left(A^{-1 / 2} B A^{-1 / 2}-m I\right)\left(M I-A^{-1 / 2} B A^{-1 / 2}\right)}{M-m} \\
& \leq \frac{1}{4}(M-m)(\ln M-\ln m) I .
\end{aligned}
$$

Multiplying both sides of (25) by $A^{1 / 2}$ we get the desired result (21).

Corollary 3. Assume that $p I \leq C \leq P I$ for some $p, P$ with $0<p<P$. Then we have that

$$
(0 \leq) \frac{p \ln p}{P-p}(P I-C)+\frac{P \ln P}{P-p}(C-p I)+\eta(C)
$$




$$
\leq \frac{\ln P-\ln p}{P-p}(C-p I)(P I-C) \leq \frac{1}{4}(P-p)(\ln P-\ln p) .
$$

\section{Further Lower ANd Upper Bounds}

We have the following result, see for instance [4]:

Lemma 2. Let $f: I \subset \mathbb{R} \rightarrow \mathbb{R}$ be a twice differentiable function on the interval $\stackrel{\circ}{I}$, the interior of $I$. If there exists the constants $d, D$ such that

$$
d \leq f^{\prime \prime}(t) \leq D \text { for any } t \in \stackrel{\circ}{I}
$$

then

$$
\begin{aligned}
\frac{1}{2} \nu(1-\nu) d(b-a)^{2} & \leq(1-\nu) f(a)+\nu f(b)-f((1-\nu) a+\nu b) \\
& \leq \frac{1}{2} \nu(1-\nu) D(b-a)^{2}
\end{aligned}
$$

for any $a, b \in \stackrel{\circ}{I}$ and $\nu \in[0,1]$.

In particular, we have

$$
\frac{1}{8}(b-a)^{2} d \leq \frac{f(a)+f(b)}{2}-f\left(\frac{a+b}{2}\right) \leq \frac{1}{8}(b-a)^{2} D
$$

for any $a, b \in \stackrel{\circ}{I}$.

The constant $\frac{1}{8}$ is best possible in both inequalities in (29).

If $D>0$, the second inequality in (28) is better than the corresponding inequality obtained by Furuichi and Minculete in [7] by applying Lagrange's theorem two times. They had instead of $\frac{1}{2}$ the constant 1 . Our method also allowed to obtain, for $d>0$, a lower bound that can not be established by Lagrange's theorem method employed in [7].

We can state the following result:

Theorem 4. Let $A, B$ be two positive invertible operators such that the condition (3) is valid, then we have

$$
\begin{aligned}
(0 \leq) \frac{1}{2 M}(B-m A) A^{-1}(M A-B) & \leq E_{m, M}(A, B) \\
& \leq \frac{1}{2 m}(B-m A) A^{-1}(M A-B) .
\end{aligned}
$$

Proof. If we consider the convex function $f(t)=t \ln t, t>0$, then $f^{\prime \prime}(t)=$ $\frac{1}{t}$ and by (19) we have

$$
\begin{aligned}
& \frac{1}{2} \nu(1-\nu) \frac{1}{\max \{a, b\}}(b-a)^{2} \\
& \leq(1-\nu) a \ln a+\nu b \ln b-((1-\nu) a+\nu b) \ln ((1-\nu) a+\nu b) \\
& \leq \frac{1}{2} \nu(1-\nu) \frac{1}{\min \{a, b\}}(b-a)^{2}
\end{aligned}
$$


for any $a, b>0$ and $\nu \in[0,1]$.

On applying the inequality (31) on the interval $[m, M]$ and for $\nu=$ $\frac{x-m}{M-m} \in[0,1]$ with $x \in[m, M]$ then we get

$$
\begin{aligned}
\frac{1}{2 M}(x-m)(M-x) & \leq \frac{M-x}{M-m} m \ln m+\frac{x-m}{M-m} M \ln M-x \ln x \\
& \leq \frac{1}{2 m}(x-m)(M-x) .
\end{aligned}
$$

Using the continuous functional calculus we have from (32) that

$$
\begin{aligned}
\frac{1}{2 M}(X-m I)(M-X I) & \leq \frac{M I-X}{M-m} m \ln m+\frac{X-m I}{M-m} M \ln M-X \ln X \\
& \leq \frac{1}{2 m}(X-m I)(M-X I)
\end{aligned}
$$

for any selfadjoint operator $X$ with the property that $m I \leq X \leq M I$.

Now, on using a similar argument to the one in the proof of Theorem 3 we deduce the desired result (30).

Finally, we have

Corollary 4. Assume that $p I \leq C \leq P I$ for some $p, P$ with $0<p<P$. Then we have the inequalities

$$
\begin{aligned}
(0 \leq) \frac{1}{2 P}(C-p I)(P I-C) & \leq \frac{p \ln p}{P-p}(P I-C)+\frac{P \ln P}{P-p}(C-p I)+\eta(C) \\
& \leq \frac{1}{2 p}(C-p I)(P I-C) .
\end{aligned}
$$

\section{Applications for Trace Inequalities}

If $\left\{e_{i}\right\}_{i \in I}$ is an orthonormal basis of $H$, we say that $A \in \mathcal{B}(H)$ is trace class provided

$$
\|A\|_{1}:=\sum_{i \in I}\left\langle|A| e_{i}, e_{i}\right\rangle<\infty .
$$

The definition of $\|A\|_{1}$ does not depend on the choice of the orthonormal basis $\left\{e_{i}\right\}_{i \in I}$. We denote by $\mathcal{B}_{1}(H)$ the set of trace class operators in $\mathcal{B}(H)$.

The following properties are also well known:

(i) We have

for any $A \in \mathcal{B}_{1}(H)$;

$$
\|A\|_{1}=\left\|A^{*}\right\|_{1}
$$

(ii) $\mathcal{B}_{1}(H)$ is an operator ideal in $\mathcal{B}(H)$, i.e.

$$
\mathcal{B}(H) \mathcal{B}_{1}(H) \mathcal{B}(H) \subseteq \mathcal{B}_{1}(H) ;
$$

(iii) $\left(\mathcal{B}_{1}(H),\|\cdot\|_{1}\right)$ is a Banach space. 
We define the trace of a trace class operator $A \in \mathcal{B}_{1}(H)$ to be

$$
\operatorname{tr}(A):=\sum_{i \in I}\left\langle A e_{i}, e_{i}\right\rangle
$$

where $\left\{e_{i}\right\}_{i \in I}$ is an orthonormal basis of $H$. Note that this coincides with the usual definition of the trace if $H$ is finite-dimensional. We observe that the series (1) converges absolutely and it is independent from the choice of basis.

The following results collects some properties of the trace:

(i) If $A \in \mathcal{B}_{1}(H)$ then $A^{*} \in \mathcal{B}_{1}(H)$ and

$$
\operatorname{tr}\left(A^{*}\right)=\overline{\operatorname{tr}(A)} \text {; }
$$

(ii) If $A \in \mathcal{B}_{1}(H)$ and $T \in \mathcal{B}(H)$, then $A T, T A \in \mathcal{B}_{1}(H)$ and

$$
\operatorname{tr}(A T)=\operatorname{tr}(T A) \text { and }|\operatorname{tr}(A T)| \leq\|A\|_{1}\|T\| ;
$$

(iii) $\operatorname{tr}(\cdot)$ is a bounded linear functional on $\mathcal{B}_{1}(H)$ with $\|\operatorname{tr}\|=1$;

(iv) $\mathcal{B}_{\text {fin }}(H)$, the space of operators of finite rank, is a dense subspace of $\mathcal{B}_{1}(H)$.

We recall that Specht's ratio is defined by [18]

$$
S(h):=\left\{\begin{array}{l}
\frac{h^{\frac{1}{h-1}}}{e \ln \left(h^{\frac{1}{h-1}}\right)} \text { if } h \in(0,1) \cup(1, \infty) \\
1 \text { if } h=1 .
\end{array}\right.
$$

It is well known that $\lim _{h \rightarrow 1} S(h)=1, S(h)=S\left(\frac{1}{h}\right)>1$ for $h>0, h \neq 1$. The function is decreasing on $(0,1)$ and increasing on $(1, \infty)$.

We consider the Kantorovich's constant defined by

$$
K(h):=\frac{(h+1)^{2}}{4 h}, h>0 .
$$

The function $K$ is decreasing on $(0,1)$ and increasing on $[1, \infty), K(h) \geq 1$ for any $h>0$ and $K(h)=K\left(\frac{1}{h}\right)$ for any $h>0$.

In the recent paper [5] we have showed amongst other that

$$
\begin{gathered}
(0 \leq) S(A \mid B)-\frac{\ln m}{M-m}(M A-B)-\frac{\ln M}{M-m}(B-m A) \leq \ln S\left(\frac{M}{m}\right) A \\
(0 \leq) S(A \mid B)-\frac{\ln m}{M-m}(M A-B)-\frac{\ln M}{M-m}(B-m A) \\
\leq \frac{4}{(M-m)^{2}}\left(K\left(\frac{M}{m}\right)-1\right)(B-m A) A^{-1}(M A-B)
\end{gathered}
$$

and

$$
\frac{1}{2 M^{2}}(B-m A) A^{-1}(M A-B)
$$




$$
\begin{aligned}
& \leq S(A \mid B)-\frac{\ln m}{M-m}(M A-B)-\frac{\ln M}{M-m}(B-m A) \\
& \leq \frac{1}{2 m^{2}}(B-m A) A^{-1}(M A-B)
\end{aligned}
$$

for positive invertible operators $A$ and $B$ that satisfy the condition (3).

Observe that, if $A, B \in \mathcal{B}_{1}(H)$ with $\operatorname{tr}(A)=\operatorname{tr}(B)=1$ and satisfy (3), then we must assume $m \leq 1 \leq M$ and by trace properties we have

$$
\begin{aligned}
\operatorname{tr}\left[(B-m A) A^{-1}(M A-B)\right] & =\operatorname{tr}\left[(m+M) B-m M A-B A^{-1} B\right] \\
& =m+M-m M-\operatorname{tr}\left(A^{-1} B^{2}\right) \\
& =(M-1)(1-m)-\chi^{2}(B, A),
\end{aligned}
$$

where $\chi^{2}(B, A)=: \operatorname{tr}\left(A^{-1} B^{2}\right)-1 \geq 0$.

We also have

$$
\frac{\ln m}{M-m}(M-1)+\frac{\ln M}{M-m}(1-m)=\ln \left(m^{\frac{M-1}{M-m}} M^{\frac{1-m}{M-m}}\right) .
$$

We can state the following result:

Proposition 1. Let $A, B \in \mathcal{B}_{1}(H)$ with $\operatorname{tr}(A)=\operatorname{tr}(B)=1$ that satisfy (3) for some $m, M$ with $0<m<1<M$. Then we have the inequalities

$$
\begin{aligned}
&(0 \leq) \operatorname{tr} S(A \mid B)-\ln \left(m^{\frac{M-1}{M-m}} M^{\frac{1-m}{M-m}}\right) \leq \ln S\left(\frac{M}{m}\right) \\
&(0 \leq) \operatorname{tr} S(A \mid B)-\ln \left(m^{\frac{M-1}{M-m}} M^{\frac{1-m}{M-m}}\right) \\
& \leq \frac{4}{(M-m)^{2}}\left(K\left(\frac{M}{m}\right)-1\right)\left[(M-1)(1-m)-\chi^{2}(B, A)\right]
\end{aligned}
$$

and

$$
\begin{gathered}
\frac{1}{2 M^{2}}\left[(M-1)(1-m)-\chi^{2}(B, A)\right] \leq \operatorname{tr} S(A \mid B)-\ln \left(m^{\frac{M-1}{M-m}} M^{\frac{1-m}{M-m}}\right) \\
\leq \frac{1}{2 m^{2}}\left[(M-1)(1-m)-\chi^{2}(B, A)\right] .
\end{gathered}
$$

Observe that

$$
\frac{m \ln m}{M-m}(M-1)+\frac{M \ln M}{M-m}(1-m)=\ln \left(m^{\frac{m(M-1)}{M-m}} M^{\frac{M(1-m)}{M-m}}\right),
$$

then by taking the trace in the inequalities (21) and (30) we can state the following result as well:

Proposition 2. Let $A, B \in \mathcal{B}_{1}(H)$ with $\operatorname{tr}(A)=\operatorname{tr}(B)=1$ that satisfy (3) for some $m, M$ with $0<m<1<M$. Then we have the inequalities

$$
(0 \leq) \ln \left(m^{\frac{m(M-1)}{M-m}} M^{\frac{M(1-m)}{M-m}}\right)-\operatorname{tr} D(A \mid B)
$$


INEQUALITIES FOR THE DUAL RELATIVE OPERATOR ENTROPY

$$
\leq \frac{\ln M-\ln m}{M-m}\left[(M-1)(1-m)-\chi^{2}(B, A)\right]
$$

and

$$
\begin{aligned}
\frac{1}{2 M}\left[(M-1)(1-m)-\chi^{2}(B, A)\right] & \leq \ln \left(m^{\frac{m(M-1)}{M-m}} M^{\frac{M(1-m)}{M-m}}\right)-\operatorname{tr} D(A \mid B) \\
& \leq \frac{1}{2 m}\left[(M-1)(1-m)-\chi^{2}(B, A)\right] .
\end{aligned}
$$

Acknowledgement. The author would like to thank the anonymous referee for valuable suggestions that have been implemented in the final version of the paper.

\section{REFERENCES}

[1] M. Klaričić Bakula, J. Pečarić, J. Perić, On the converse Jensen inequality, Appl. Math. Comp. 218 (2012), 6566-6575.

[2] S. S. Dragomir, Bounds for the normalized Jensen functional, Bull. Austral. Math. Soc. 74 (3)(2006), 417-478.

[3] S. S. Dragomir, A note on Young's inequality, Rev. R. Acad. Cienc. Exactas Fís. Nat. Ser. A Math. RACSAM 111 (2017), no. 2, 349-354. Preprint RGMIA Res. Rep. Coll. 18 (2015), Art. 126. [http://rgmia.org/papers/v18/v18a126.pdf] .

[4] S. S. Dragomir, A note on new refinements and reverses of Young's inequality, Transylv. J. Math. Mech. 8 (2016), no. 1, 45-49. Preprint RGMIA Res. Rep. Coll. 18 (2015), Art. 131. [http://rgmia.org/papers/v18/v18a131.pdf] .

[5] S. S. Dragomir, Some inequalities for relative operator entropy, Preprint RGMIA Res. Rep. Coll. 18 (2015), Art. 145 [http://rgmia.org/papers/v18/v18a145.pdf] .

[6] S. Furuichi, Precise estimates of bounds on relative operator entropies, Math. Ineq. Appl. 18 (2015), 869-877.

[7] S. Furuichi and N. Minculete, Alternative reverse inequalities for Young's inequality, J. Math Inequal. 5 (2011), Number 4, 595-600.

[8] J. I. Fujii and E. Kamei, Uhlmann's interpolational method for operator means. Math. Japon. 34 (1989), no. 4, 541-547.

[9] J. I. Fujii and E. Kamei, Relative operator entropy in noncommutative information theory. Math. Japon. 34 (1989), no. 3, 341-348.

[10] T. Furuta, J. Mićić Hot, J. Pečarić and Y. Seo, Mond-Pečarić Method in Operator Inequalities. Inequalities for bounded selfadjoint operators on a Hilbert space. Monographs in Inequalities, 1. Element, Zagreb, 2005. xiv+262 pp.+loose errata. ISBN: 953-197-572-8.

[11] R. Jakšić, J. Pečarić, On some new converses of convex inequalities in Hilbert space, Banach J. Math. Anal. b (2), (2015), 63-82.

[12] I. H. Kim, Operator extension of strong subadditivity of entropy, J. Math. Phys. 53(2012), 122204

[13] P. Kluza and M. Niezgoda, Inequalities for relative operator entropies, Elec. J. Lin. Alg. 27 (2014), Art. 1066.

[14] D. S. Mitrinović, ,J. E. Pečarić, A. M. Fink, Classical and new inequalities in analysis. Mathematics and its Applications, East European Series, 61. Kluwer Academic Publishers Group, Dordrecht, 1993. 
[15] M. S. Moslehian, F. Mirzapour, and A. Morassaei, Operator entropy inequalities. Colloq. Math., 130 (2013), 159-168.

[16] M. Nakamura and H. Umegaki, A note on the entropy for operator algebras. Proc. Japan Acad. 37 (1961) 149-154.

[17] I. Nikoufar, On operator inequalities of some relative operator entropies, Adv. Math. 259 (2014), 376-383.

[18] W. Specht, Zer Theorie der elementaren Mittel, Math. Z. 74 (1960), pp. 91-98.

Silvestru Sever Dragomir

Victoria UniVERSiTy,

College of Engineering \& Science,

PO Box 14428, Melbourne City, MC 8001, Australia

Email address: sever.dragomir@vu.edu.au 